\title{
Cocaine-induced locomotor activation differs across six sets of inbred mouse substrains
}

1 Christiann H. Gaines ${ }^{1,2+}$, Sarah A. Schoenrock ${ }^{1+}$, Joseph Farrington ${ }^{1}$, David F. Lee ${ }^{1,3}$, Lucas J. 2 Aponte-Collazo ${ }^{3,4}$, Ginger Shaw ${ }^{1}$, Darla R. Miller ${ }^{1,5}$, Martin T. Ferris ${ }^{1}$, Fernando Pardo3 Manuel de Villena ${ }^{1,5}$ and Lisa M. Tarantino ${ }^{1,6}$

$4{ }^{1}$ Department of Genetics, School of Medicine, University of North Carolina at Chapel Hill, NC, 5 United States

$6 \quad{ }^{2}$ Neuroscience Curriculum, University of North Carolina at Chapel Hill, NC, United States

$7 \quad{ }^{3}$ Pharmacology Curriculum, University of North Carolina at Chapel Hill, NC, United States

$8{ }^{4}$ Department of Pharmacology, School of Medicine, University of North Carolina at Chapel Hill, NC,

9 United States

$10{ }^{5}$ Lineberger Comprehensive Cancer Center, School of Medicine, University of North Carolina at

11 Chapel Hill, NC, United States

$12{ }^{6}$ Division of Pharmacotherapy and Experimental Therapeutics, Eshelman School of Pharmacy,

13 University of North Carolina at Chapel Hill, NC, United States

$14{ }^{+}$Denotes joint first-authorship, authors contributed equally

\section{5 *Correspondence: Lisa M. Tarantino}

16 Corresponding Author

17 lisat@med.unc.edu

18 Keywords: cocaine sensitivity, initial cocaine response, genetics, reduced complexity cross, rodent 19 behavior, addiction, rodent model, mice models 


\section{Abstract}

33 Cocaine use disorders (CUD) are devastating for affected individuals and impose a significant 34 burden on society, but there are currently no FDA-approved therapies. The development of novel and 35 effective treatments has been hindered by substantial gaps in our knowledge about the etiology of these 36 disorders. The risk for developing a CUD is influenced by genetics, the environment and complex 37 interactions between the two. Identifying specific genes and environmental risk factors that increase 38 CUD risk would provide an avenue for the development of novel treatments.

39 Rodent models of addiction-relevant behaviors have been a valuable tool for studying the genetics of response to drugs of abuse. Traditional genetic mapping using genetically and phenotypically divergent inbred mice has been successful in identifying numerous chromosomal regions that influence addiction-relevant behaviors, but these strategies rarely result in identification of the causal gene or genetic variant. To overcome this challenge, reduced complexity crosses (RCC) between closely related inbred mouse substrains have been proposed as a method for rapidly identifying and validating functional variants. The RCC approach is dependent on identifying phenotypic differences between substrains. To date, however, the study of addiction-relevant behaviors has been limited to very few sets of substrains, mostly comprising the C57BL/6 lineage.

The present study expands upon the current literature to assess cocaine-induced locomotor activation in 20 inbred mouse substrains representing six inbred strain lineages (A/J, BALB/c, FVB/N, $\mathrm{C} 3 \mathrm{H} / \mathrm{He}, \mathrm{DBA} / 2$ and NOD) that were either bred in-house or supplied directly by a commercial vendor. To our knowledge, we are the first to identify significant differences in cocaine-induced locomotor response in several of these inbred substrains. The identification of substrain differences allows for the initiation of RCC populations to more rapidly identify specific genetic variants associated with acute cocaine response. The observation of behavioral profiles that differ between mice generated in-house and those that are vendor-supplied also presents an opportunity to investigate the influence of environmental factors on cocaine-induced locomotor activity. 


\section{Introduction}

64 Cocaine remains the second most commonly used drug worldwide and both cocaine use and cocaine related overdose deaths have been increasing in the United States in recent years (Karila, Petit et al. 2012, Jalal, Buchanich et al. 2018, Bentzley, Han et al. 2021). Over half of cocaine users in the United States have a diagnosed cocaine use disorder (CUD), indicating the high addiction liability of cocaine (Goldman, Oroszi et al. 2005, SAMHSA 2019). Despite the prevalence of CUD, there are currently no FDA approved therapies. The lack of treatment options is partially due to gaps in our knowledge about the etiology of this complex and devastating disorder.

Not all who use cocaine will go on to develop a CUD, suggesting that there are individual differences in risk. The risk of developing a CUD is influenced by genetics, the environment and interactions between the two (Merikangas and Avenevoli 2000, Goldman, Oroszi et al. 2005, Thatcher and Clark 2008, Ducci and Goldman 2012, Mennis, Stahler et al. 2016). Twin studies suggest heritability estimates of approximately 0.70 for cocaine dependence indicating a significant contribution of genetics (Ducci and Goldman 2012). Human genome wide association studies (GWAS) are currently being conducted to identify specific loci and genes associated with substance use disorders (Sullivan, Agrawal et al. 2018) and have been highly successful for some drugs of abuse such as nicotine and alcohol. However, very few GWAS studies have been published for cocaine dependence or CUD (Gelernter, Sherva et al. 2014, Cabana-Dominguez, Shivalikanjli et al. 2019, Sun, Kranzler et al. 2020) due to lack of sufficient sample size of individuals with cocaine dependence for current GWAS methods, which has limited the genetic discovery in GWAS for specific loci and genes. Identifying genes and molecular pathways that increase CUD risk would provide insight into individuals at increased risk and novel targets that could be investigated for the development of therapeutics. genetic mapping studies and mechanistic follow-up of GWAS loci in rodent models. A notable example is the replication of the identification of family with sequence similarity 53 , member B $(F A M 53 B)$, as a risk variant for cocaine dependence in a mouse mapping population for selfadministration of cocaine (Gelernter, Sherva et al. 2014, Dickson, Miller et al. 2016). The use of rodent models offers several advantages, including the ability for the genetic background, environment, and recapitulate the range of symptoms observed in humans with CUD, they do allow for measurement of specific addiction-relevant behaviors, including initial drug sensitivity. Retrospective and longitudinal 
predict subsequent drug use (Haertzen, Kocher et al. 1983, Davidson, Finch et al. 1993, Lambert, McLeod et al. 2006, de Wit and Phillips 2012). In mice, acute locomotor response to an initial dose of cocaine is a well-established model of initial sensitivity (Thomsen and Caine 2011, Wiltshire, Ervin et al. 2015).

Traditional genetic mapping approaches using inbred mouse strains have been used successfully to identify genomic regions, termed quantitative trait loci (QTL), that are associated with cocaine-induced locomotor activation (Tolliver, Belknap et al. 1994, Miner and Marley 1995, Phillips, Huson et al. 1998, Jones, Tarantino et al. 1999, Boyle and Gill 2001, Gill and Boyle 2003, Boyle and Gill 2009, Dickson, Miller et al. 2016). Mapping approaches involve outcrosses between genetically and phenotypically diverse pairs of inbred strains and the resulting F1s are intercrossed or backcrossed to generate F2 or N2 mapping populations, respectively. The resulting QTL identified from a F2 or N2 population typically span tens of megabases and comprise hundreds of genes and thousands of potential causal polymorphisms. Therefore, identifying the causative variants that affect cocaine-induced locomotor activation and other complex behavioral traits has been extremely challenging.

The use of Reduced Complexity Crosses (RCC) for genetic mapping offers a significant advantage over traditional genetic mapping strategies. Mouse substrains are nearly isogenic inbred mouse substrains that are derived from the same founder inbred strain but have been bred independently for many generations (typically more than 20). An RCC is generated in the same fashion as an F2 or N2 population described above, using two substrains that differ for a phenotype of interest. The size of the resulting QTL confidence interval is similar to a traditional F2 mapping population, spanning ten(s) of megabases. However, within a QTL interval, the two nearly isogenic substrains will have significantly fewer variants, limited to polymorphisms that were either segregating at the time the strains were separated or arose spontaneously after that time (Bryant CD 2018). This feature dramatically facilitates follow-up on the few polymorphisms within the QTL and identification of the causative polymorphism. Additionally, a recently developed genotyping array captures polymorphisms between inbred mouse substrains, facilitating rapid and reliable genotyping of RCCs (Sigmon, Blanchard et al. 2020). A RCC has been used successfully to identify a genetic polymorphism in the Cyfip 2 gene that results in differing psychostimulant responses in C57BL/6J and C57BL/6N substrains (Kumar, Kim et al. 2013).

123 Genetic differences between substrains are likely to influence any number of phenotypes, offering a

124 powerful tool with which to expand our knowledge about the genetic loci that affect addiction-relevant 125 behaviors. Thus far, the literature describing substrain differences in locomotor response to drugs of 126 abuse has focused on C57BL/6 substrains. In this study, we measured cocaine-induced locomotor 
127 activation across 6 substrains derived from A/J, BALB/c, DBA/2, FVB/N, NOD and C3H/He inbred 128 mouse strains. We report significant differences across these strains within their respective substrains

129 for acute cocaine sensitivity. These data significantly expand knowledge about substrain differences in 130 cocaine locomotor response and offer the opportunity to pursue genetic studies to identify genes that 131 contribute to this behavior.

\section{Materials and Methods}

\subsection{General Methods}

135 Mice were all housed in a pathogen-free facility at UNC. This facility consisted of a 12-hour light/dark cycle with lights on at 7:00 AM. All animal care and protocols were approved by the The

137 University of North Carolina at Chapel Hill (UNC) Institutional Animal Care and Use Committees and 138 followed guidelines that were implemented by the National Institutes of Health Guide for the Care and 139 Use of Laboratory Animals., $8^{\text {th }}$ Edition. Mice were maintained in AAALAC-accredited, specific 140 pathogen free (SPF) barrier colony in ventilated cages (Tecniplast, Buguggiate, Italy). Food (PicoLab 141 Rodent Diet 20, Purina, St. Louis, Missouri) was provided ad libitum and throughout the duration of 142 behavioral testing. Edstrom carbon filtered; reverse osmosis hyper-chlorinated water was provided ad 143 libitum except during behavioral testing.

144 Two groups of mice were used for behavioral testing (see Supp Table 1 for a summary of 145 substrains, origin, housing, and vendor). The first group consisted of five sets of substrains that were 146 purchased directly from their respective commercial vendors and were then group housed by substrain 147 and sex. The second group consisted of five sets of substrains that were originally purchased from their 148 respective vendors, but mice tested in this study were bred in our laboratory at UNC. Mice bred at 149 UNC were either group-housed with cagemates of the same substrain or co-housed with mice from 150 other substrains within their strain group (i.e. DBA/2J, DBA/2NCrl and DBA/2NTac mice in the same 151 cage). Mice were co-housed at weaning, around postnatal day 21.

152 Vendor supplied substrains were an average age of 62 days old at the start of testing. In-house bred 153 mice were an average age of 65 days old at the start of testing. All mice were weighed on the day prior 154 to testing and weights were used to determine the volume of saline or cocaine administered during 155 open field testing. On the day of testing, mice were transported to the testing room, which was located 156 within the same vivarium, immediately prior to the start of testing. Behavioral testing occurred during 157 the light cycle from 8:00 AM to 12:00 PM with the time that a mouse was tested being consistent across 158 the three test days. 


\subsection{Animals}

\subsubsection{Vendor Supplied Substrains}

$\mathrm{A} / \mathrm{J}, \mathrm{BALB} / \mathrm{c}, \mathrm{FVB} / \mathrm{N}$ and DBA/2 substrains were purchased from their respective commercial vendors and housed in substrain specific cages throughout testing. Mice were an average of 27 days old upon arrival to UNC and were acclimated to the vivarium for 5 weeks after arrival before behavioral testing. A/J substrains were A/J (The Jackson Laboratory, 000646), A/JCr (Charles River Laboratories, 563) and A/JOlaHsd (Envigo, 049). BALB/c substrains were BALB/cJ (The Jackson Laboratory, 000651), BALB/cByJ (The Jackson Laboratory, 001026), BALB/cAnNCrl (Charles River Laboratories, 028), BALB/cAnNHsd (Envigo, 047). FVB/N substrains were FVB/NJ (The Jackson Laboratory, 001800), FVB/NCrl (Charles River Laboratories, 207), FVB/NHsd (Envigo, 118), and FVB/NTac (Taconic Biosciences, FVB-F/FVB-M). DBA/2 substrains were DBA/2J (The Jackson Laboratory, 000671), DBA/2NCrl (Charles River Laboratories, 026) and DBA/2NTac (Taconic Biosciences, DBA2-F/DBA2-M).

\subsubsection{Substrains Bred In-House}

Another cohort of inbred mouse substrains were purchased from commercial vendors but test animals were bred in-house at UNC. The following substrains were tested in this cohort: DBA/2J, DBA/2NCrl, DBA/2NTac, A/J, A/JOlaHsd, BALB/cByJ, BALB/cJ, FVB/NJ, FVB/NTac, NOD/MrkTac (Taconic Biosciences, NOD-F/NOD-M), NOD/ShiLtJ (The Jackson Laboratory, \#001976), C3H/HeJ (The Jackson Laboratory, \#000659), C3H/HeNTac (Taconic Biosciences, C3HF/C3H-M), C3H/HeNHsd (Envigo, 040) and C3H/HeNCrl (Charles River Laboratories, 025). Some of these mice were cohoused with cagemates that included at least two different substrains from a single progenitor strain. Information on all inbred substrains including cage environment (cohoused vs not cohoused) is provided in Supplemental Table 1.

\subsection{Drugs}

184 Cocaine hydrochloride $(\mathrm{HCl})$ was purchased from Sigma-Aldrich (St. Louis, MO, USA; C5776185 5G). A solution of cocaine $\mathrm{HCl}$ was prepared fresh daily. Cocaine $\mathrm{HCl}$ was dissolved in physiological saline at a concentration of $2 \mathrm{mg} / \mathrm{ml}$ and administered via intraperitoneal (i.p.) injection at a volume of $0.01 \mathrm{ml} / \mathrm{g}$ resulting in a dose of $20 \mathrm{mg} / \mathrm{kg}$ of body weight administered to mice for behavioral testing.

188 Saline was purchased from Fisher Scientific (Waltham, MA, USA; 297753). 
The open field (OF) arena (ENV-515-16, Med Associates, St. Albans, VT, USA), measured $17 \times 17 \times 13 \mathrm{~cm}$ and consisted of four clear Plexiglas walls and a white Plexiglas floor. The walls are surrounded by infrared detection beams on the $\mathrm{X}, \mathrm{Y}$ and $\mathrm{Z}$ axes used to detect horizontal and vertical activity of the animal throughout the duration of the test session. The OF chamber is placed within a sound attenuating box $(73.5 \times 59 \times 59 \mathrm{~cm})$ that has two overhead light fixtures containing $28-\mathrm{V}$ lamps. Light levels on the arena floor were 24 lux in the center, 10 lux in the corners and 13 lux along the walls. Eight identical OF arenas were used for testing with a mouse being tested in the same arena each test day.

\subsection{Acute Cocaine-Induced Locomotor Activity Test}

On day 1 (habituation) and 2 (baseline), mice were given an i.p. injection of saline at a volume of $0.01 \mathrm{ml} / \mathrm{g}$ body weight and immediately placed into the OF chambers for $30 \mathrm{mins}$. On day 3 (acute cocaine exposure), mice were given an i.p. injection of $20 \mathrm{mg} / \mathrm{kg}$ of cocaine and placed into the $\mathrm{OF}$ chamber for 30 mins. At the end of each test session, mice were placed back into their home cages and the OF chambers were cleaned with $0.25 \%$ bleach. Locomotor behavior was measured as total distance moved (in centimeters) for the entire 30-min test period each day using the manufacturers data acquisition software (Activity Monitor v5.9.725; Med Associates).

\subsection{Statistical Analysis}

All statistical analyses were performed using SPSS v28 for Mac (IBM Inc). For each set of substrains, we performed an ANOVA that included day of testing, substrain and sex as independent variables and locomotor activity as the dependent variable. Significant main effects $(p<0.05)$ were

212 followed up with post-hoc Tukey’s HSD or independent samples T-tests.

\section{Results}

215 Experimental data for all inbred mouse substrains including origin of the mice, number of mice

216 tested, cage environment, strain means and standard deviations are provided in Supplemental Table

217 1. We observed significant substrain differences in basal and/or cocaine-induced locomotor activity

218 in 4 of the 6 strain groups we examined. $\mathrm{C} 3 \mathrm{H} / \mathrm{He}$ and DBA/2 substrain differences were fairly stable 219 across experimental cohorts. We also observed substrain differences (i.e. A/J and FVB/N) that were 220 not replicated across experimental groups (vendor-supplied vs in-house). Sex differences also varied 221 across and within strain groups and experimental cohorts. Results presented individually by substrain 222 are described below. 


\subsection{A/J substrain behavior differs across experimental cohorts}

\section{Vendor Supplied}

Overall, the locomotor activity of the vendor-supplied A/J substrains did not increase

significantly after exposure to cocaine $\left(\mathrm{F}_{(2,125)}=0.82 ; p=0.445\right)$, although increases in cocaineinduced locomotor activity can be observed in $\mathrm{A} / \mathrm{JCr}$ and $\mathrm{A} / \mathrm{JOlaHsd}$ substrains (Fig 1A). There was a significant main effect of substrain $\left(\mathrm{F}_{(2,125)}=5.5 ; p=0.005\right)$. $\mathrm{A} / \mathrm{JCr}$ mice were significantly more active than either $\mathrm{A} / \mathrm{J}(p=0.047)$ and $\mathrm{A} / \mathrm{JOlaHsd}$ mice $(p=0.006)$. No significant sex $\left(\mathrm{F}_{(1,125)}=0.05\right.$; $p=0.827$ ) or interaction effects were observed.

\section{Bred In-House}

Two A/J substrains were bred in-house at UNC for 1-2 (A/JOlaHsd) or 3-4 (A/J) generations.

These mice showed a dramatically different behavioral profile than those obtained from commercial vendors (Fig 1B). We observed a significant decrease in locomotor activity across all three days $\left(\mathrm{F}_{(2,207)}=17.8 ; \mathrm{p}=6.9 \times 10^{-8}\right)$. We also observed significant substrain $\left(\mathrm{F}_{(1,207)}=26.1 ; p=7.4 \times 10^{-7}\right)$ and sex $\left(\mathrm{F}_{(1,207)}=8.6 ; p=0.004\right)$ effects. The $\mathrm{A} / \mathrm{J}$ substrain showed significantly higher locomotor activity across all three days $\left(t(208)=4.7 ; p=5.0 \times 10^{-6}\right)$ and males were significantly more active than females $(t(182)=2.5 ; p=0.012)$. There were no significant interactions among any of the independent variables tested.

\subsection{Locomotor response to cocaine differs in BALB/c substrains from the " $\mathrm{J}$ " lineage in}

\section{Vendor Supplied}

We observed significant changes in locomotor activity in the four vendor-supplied BALB/c substrains across three days of testing $\left(\mathrm{F}_{(2,162)}=7.7 ; p=6.2 \times 10^{-4}\right)$. Collapsed across strains, locomotor activity in response to cocaine on Day 3 is significantly higher than Day $2(\mathrm{p}=0.006)$ but not Day $1(\mathrm{p}=0.889)$ activity in response to saline. We also observed a significant substrain $\left(\mathrm{F}_{(3,162)}\right.$ $\left.=13.7 ; p=5.0 \times 10^{-8}\right)$ and substrain $\mathrm{x}$ day interaction $\left(\mathrm{F}_{(6,162)}=2.7 ; p=0.017\right)($ Fig 2$)$. Mice of both $\mathrm{J}$ substrains (BALB/cJ and BALB/cByJ) are significantly more active than BALB/cAnNHsd mice on Day 1 (both $p<0.05$ ). Although mice of both $\mathrm{J}$ substrains are more active than BALB/cAnNCrl mice on Day 1, the difference is only significant for BALB/cJ mice $(p<0.05)$. The same general pattern is observed for locomotor activity on Day 2 in the $\mathrm{J}$ strains vs BALB/cAnNCrl, but neither J substrain differs from BALB/cAnNHsd. Finally, on Day 3 BALB/cByJ mice are significantly more active in 
0.01). Female mice were significantly more active than male mice across all days and collapsed across substrain $(t(184)=-2.0 ; p=0.023)$.

\section{Bred In-House}

$\mathrm{BALB} / \mathrm{cJ}$ and BALB/cByJ mice were generated in-house and offspring from the $1^{\text {st }}$ and $2^{\text {nd }}$ generations of breeding from the initial vendor stock. We observed no significant day $\left(\mathrm{F}_{(2,27)}=0.87\right.$; $\mathrm{p}=0.431)$ or substrain $\left(\mathrm{F}_{(1,27)}=0.01 ; \mathrm{p}=0.927\right)$ differences (data not shown). We also observed no sex differences $\left(\mathrm{F}_{(1,27)}=2.3 ; \mathrm{p}=0.145\right)$ but it should be noted that our experimental cohort was limited to only 2 BALB/cByJ males and no BALB/cJ males.

\subsection{Basal and cocaine-induced locomotor differences are consistent across different} experimental cohorts of $\mathrm{C} 3 \mathrm{H} / \mathrm{He}$ substrains

\section{Bred in-house}

All C3H/He substrains were bred in-house. The initial cohort was limited to $\mathrm{C} 3 \mathrm{H} / \mathrm{HeJ}$ and $\mathrm{C} 3 \mathrm{H} / \mathrm{HeNTac}$ substrains and were the first generation of offspring from vendor-supplied mice $(\mathrm{C} 3 \mathrm{H} / \mathrm{HeJ})$ or offspring of crosses between mice from the third generation bred at UNC addition to $\mathrm{C} 3 \mathrm{H} / \mathrm{HeNTac}$ and $\mathrm{C} 3 \mathrm{H} / \mathrm{HeJ}$, and were produced by breeding vendor-supplied mice at UNC for one generation.

Both $\mathrm{C} 3 \mathrm{H} / \mathrm{HeJ}$ and $\mathrm{C} 3 \mathrm{H} / \mathrm{HeNTac}$ substrains in the first cohort were significantly more active in response to cocaine (Day 3 ) vs saline (Days 1 and 2$)\left(F_{(2,105)}=216.0 ; p=8.3 \times 10^{-46}\right)$. We also observed a significant substrain effect $\left(\mathrm{F}_{(1,105)}=39.3 ; \mathrm{p}=8.2 \times 10^{-9}\right)$ and substrain $\mathrm{x}$ day interaction $\left(\mathrm{F}_{(2,105)}=15.3 ; p=1.0 \times 10^{-6}\right) . \mathrm{C} 3 \mathrm{H} / \mathrm{HeNTac}$ mice were significantly more active than $\mathrm{C} 3 \mathrm{H} / \mathrm{HeJ}$ mice in response to saline (Fig 3A) on Days $1\left(t(37)=-5.7 ; p=2.0 \times 10^{-6}\right)$ and $2(t(37)=-4.2 ; p=1.6 \mathrm{x}$ $10^{-4}$ ) and in response to cocaine on Day $3\left(t(29)=-5.1 ; p=1.8 \times 10^{-5}\right)$. No sex differences were observed $\left(\mathrm{F}_{(1,105)}=2.3 ; p=0.132\right)$.

In the second cohort comparing all four $\mathrm{C} 3 \mathrm{H} / \mathrm{He}$ substrains, we observed a significant effect of substrain $\left(\mathrm{F}_{(3,528)}=32.7 ; \mathrm{p}=2.2 \times 10^{-19}\right)$ and day $\left(\mathrm{F}_{(2,528)}=467.6 ; p=1.4 \times 10^{-117}\right)$ and a substrain by day interaction $\left(\mathrm{F}_{(6,528)}=8.0 ; p=2.9 \times 10^{-8}\right)$. As in the first cohort, $\mathrm{C} 3 \mathrm{H} / \mathrm{HeNTac}$ mice were significantly more active than $\mathrm{C} 3 \mathrm{H} / \mathrm{HeJ}\left(p=5.1 \times 10^{-13}\right)$ and both of the additional $\mathrm{C} 3 \mathrm{H} / \mathrm{He}$ substrains (Fig 3B; all $p<0.001)$. No sex differences were observed $\left(\mathrm{F}_{(1,528)}=0.031 ; p=0.86\right)$. 


\subsection{Vendor-supplied and in-house DBA/2 substrains show strikingly similar differences in} basal and cocaine-induced locomotor behavior

\section{Vendor Supplied}

We observed significant day $\left(\mathrm{F}_{(2,126)}=23.0 ; p=3.1 \times 10^{-9}\right)$ and substrain $\left(\mathrm{F}_{(2,126)}=13.4 ; p=\right.$ $5.0 \times 10^{-6}$ ) differences among the three vendor-supplied DBA/2 substrains - DBA/2J, DBA/2NTac and $\mathrm{DBA} / 2 \mathrm{NCrl}$. There was also a significant day $\mathrm{x}$ substrain interaction $\left(\mathrm{F}_{(4,126)}=3.4 ; p=0.011\right)$. $\mathrm{DBA} / 2 \mathrm{NTac}$ mice were significantly less active than DBA/2NCrl mice on Day $1(p=0.04)$ and both DBA/2NCrl and DBA/2J mice on Days 2 (all $p<0.01)$ and 3 (all $p<0.05$ ). No significant sex differences were observed (Fig 4A).

\section{Bred In-House}

The same set of DBA/2 mice were bred in-house at UNC and mice from the first generation were tested for cocaine-induced locomotor activity. We observed a similar pattern of behavior in these DBA/2 substrains. There were significant day $\left(\mathrm{F}_{(2,288)}=48.1 ; p=9.1 \times 10^{-19}\right)$ and substrain $\left(\mathrm{F}_{(2,288)}=\right.$ $\left.11.0 ; p=2.4 \times 10^{-5}\right)$ effects and a significant day $\mathrm{x}$ substrain interaction $\left(\mathrm{F}_{(4,288)}=7.7 ; p=6.0 \times 10^{-6}\right)$. Although none of the substrains differed for locomotor activity on Day 1, DBA/2NTac mice had significantly lower locomotor activity than DBA/2J mice on Day $2(p=0.018)$ and both DBA/2J $(p=$ $\left.4.6 \times 10^{-4}\right)$ and DBA/2NCrl mice $(p=0.003)$ on Day $3($ Fig 4B).

\subsection{Basal and cocaine-induced locomotor behavior differs across FVB/N mice bred in-house}

\section{$309 \quad$ Vendor Supplied}

310 We observed no significant substrain $\left(\mathrm{F}_{(3,72)}=0.76 ; p=0.519\right)$ or $\operatorname{sex}\left(\mathrm{F}_{(1,72)}=0.03 ; p=0.859\right)$

differences and no significant interaction effects among the four vendor-supplied FVB/N substrains -

312 FVB/NJ, FVB/NTac, FVB/NCrl and FVB/NHsd. However, all FVB/N substrains showed

313 significantly increased locomotor activity on Day 3 after exposure to cocaine $\left(\mathrm{F}_{(2,72)}=123.2 ; p=\right.$

$3145.7 \times 10^{-24} ;$ Fig 5A).

\section{Bred In-House}

317 Locomotor activity in response to cocaine was significantly higher than response to saline in both 318 of the FVB/N substrains that were bred in-house for 1-2 generations - FVB/NJ and FVB/NTac

$319\left(\mathrm{~F}_{(2,108)}=31.3 ; \mathrm{p}=1.9 \times 10^{-11} ; \mathrm{Fig} 5 \mathrm{5B}\right) . \mathrm{FVB} / \mathrm{NTac}$ mice exhibited higher locomotor activity across all 320 three days in comparison to $\mathrm{FVB} / \mathrm{NJ}$ mice $(t(118)=-2.8 ; p=0.007)$. There was no significant main 
321 effect of sex, but we did observe a significant strain x sex interaction. FVB/NTac males were

322 significantly more active than FVB/NJ males $(t(42)=-3.0 ; p=0.005)$ but FVB/NTac and FVB/NJ

323 females did not differ $(t(64)=0.60 ; p=0.552)$.

\subsection{Locomotor response to cocaine did not differ across 2 NOD substrains}

326 Bred In-House

327 NOD substrain testing was limited to those that were bred in-house at UNC. We observed a

328 significant effect of day with both NOD/ShiLtJ and NOD/MrkTac showing a robust increase in

329 locomotor activity on Day 3 after exposure to cocaine (Fig 6; $\mathrm{F}_{(2,240)}=177.9 ; p=4.2 \times 10^{-48}$ ).

330 NOD/ShiLtJ mice were slightly less active on Days 1 and 2, but this difference was not significant

$331\left(\mathrm{~F}_{(1,240)}=3.0 ; p=0.084\right)$. No significant strain, sex or interaction effects were observed.

\subsection{Discussion}

Laboratory mice are invaluable tools in biomedical research and have contributed greatly to our understanding of biological and disease processes. Inbred strains, in particular, have been used for decades in genetic studies aimed at identifying genes and genetic loci that contribute to behavioral phenotypes including responses to various drugs of abuse (Tarantino, McClearn et al. 1998, Jones, Tarantino et al. 1999, Philip, Duvvuru et al. 2010, Yazdani, Parker et al. 2015). While these studies have been very successful in identifying chromosomal regions that likely harbor causal genetic variants, the genetic diversity present in mapping crosses between two standard inbred strains has hindered progress and these strategies have rarely progressed to identifying a specific causal gene or variant. The reduced genetic complexity in inbred mouse substrains offers the opportunity to overcome this hurdle and more rapidly and efficiently identify both the causative gene and specific genetic variant.

In order to use the RCC approach to identify causative genes and genetic variants one needs to identify substrains that exhibit phenotypic differences in the trait of interest. For example, this approach has been used successfully to identify the Cyfip2 gene as a regulator of basal and cocaine-induced locomotor activity, behavioral sensitization and binge-eating in two C57BL/6 substrains, C57BL/6J and C57BL/6NJ (Kumar, Kim et al. 2013)(Kirkpatrick, Goldberg et al. 2017). The study of addictionrelated behaviors, and specifically initial locomotor sensitivity to psychostimulants, has been mostly limited to the C57BL/6 inbred substrains. We assessed differences in cocaine-induced locomotor response across 20 inbred mouse substrains from 6 different strain sets. Thus, our data represent the first characterization of cocaine-induced locomotor activation in most of these substrains. 
Two sets of strains showed particularly robust substrain differences that were replicated across experimental cohorts. $\mathrm{C} 3 \mathrm{H} / \mathrm{HeNTac}$ mice had significantly higher basal and cocaine-induced locomotor activity than $\mathrm{C} 3 \mathrm{H} / \mathrm{HeNCrl}$ and $\mathrm{C} 3 \mathrm{H} / \mathrm{HeNHsd}$ mice in one experimental cohort and $\mathrm{C} 3 \mathrm{H} / \mathrm{HeJ}$ mice in both cohorts (Fig 3). The similarity of the behavioral phenotype in $\mathrm{C} 3 \mathrm{H} / \mathrm{HeJ}, \mathrm{C} 3 \mathrm{H} / \mathrm{HeNCrl}$ and $\mathrm{C} 3 \mathrm{H} / \mathrm{HeNHsd}$ substrains suggests that the causal variant or variants became fixed in the $\mathrm{C} 3 \mathrm{H} / \mathrm{HeNTac}$ substrain after it diverged from the other $\mathrm{C} 3 \mathrm{H} / \mathrm{HeN}$ lines in $1974(\mathrm{C} 3 \mathrm{H} / \mathrm{HeNCrl})$ and 1983 (C3H/HeNHsd). However, we must also consider the possibility that $\mathrm{C} 3 \mathrm{H} / \mathrm{HeNCrl}$ and $\mathrm{C} 3 \mathrm{H} / \mathrm{HeNHsd}$ behavioral phenotypes result from a different variant or variants. We also observed consistent substrain differences in behavior in DBA/2 mice that were supplied by commercial vendors and and bred in-house. DBA/2NTac mice had significantly lower basal and cocaine-induced locomotor activity in comparison with DBA/2J and DBA/2NCrl in both cohorts (Fig 4). These data suggest that the causal variant(s) likely arose in the DBA/2NTac substrain after it diverged from DBA/2N in 1981.

Correspondence of significant substrain differences across the two cohorts suggests a strong genetic component and supports the use of the RCC to identify specific causal variants that influence basal locomotor activity and response to cocaine. We do note that we observed significant substrain differences in either basal or cocaine-induced locomotor behavior in 4 out of the 6 strain sets, suggesting that multiple genes likely contribute to these behaviors and more than one variant may be responsible for behavioral differences observed in any set of substrains. Therefore, larger mapping cohorts may be required for QTL identification (Bryant CD 2018), but the advantages of using a RCC for variant identification still apply.

We also identified different behavioral phenotypes in vendor-supplied substrains vs those bred inhouse. For example, FVB/NTac mice bred in-house were significantly more active than FVB/NJ mice bred in-house across all three days of testing whereas vendor-supplied FVB substrains showed similar locomotor behavior across all three test days (Figs 5A, B). Neither A/J nor A/JOlaHsd substrains bred in-house were significantly activated in response to cocaine and in fact, locomotor behavior in these two substrains decreased significantly across all three days of testing (Fig 1B). Vendor-supplied A/J and $\mathrm{A} / \mathrm{JOlaHsd}$ substrains were also not significantly activated in response to cocaine, but locomotor behavior was similar across test days with no significant decrease in activity (Fig 1A).

The observation of behavioral differences in the same substrain based on the source from which mice were obtained suggests that environmental factors could be responsible. Multiple studies have systematically examined environmental factors that might affect behavioral phenotypes including, but 
Wahlsten, Metten et al. 2003, Sorge, Martin et al. 2014). However, previous studies have generally assessed behavioral differences in mice tested across multiple sites. We examined behavior in all mice, independent of the source, in the same behavioral facility (and same testing room) at UNC. As such, we were able to control, to the extent possible, the environment to which the mice were exposed in the 5-week period leading up to testing. Mice were maintained on the same light cycle, tested during the same time of day, provided the same diet and water and housed in the same caging and animal holding room prior to and throughout testing.

The stress of transportation is an obvious difference between vendor-supplied mice and those bred in-house. We don't believe transportation stress could fully explain observed behavioral differences between mice from different sources. Previous studies have shown that transportation has very little effect on behavioral outcomes (Crabbe, Wahlsten et al. 1999, Chesler, Wilson et al. 2002). Moreover, vendor-supplied mice arrived at UNC very close to weaning age and were habituated to our vivarium conditions for approximately 5 weeks prior to testing.

Experimenter effects may have also had an impact on behavioral outcomes. All mice supplied directly from the vendor were tested by the same animal handler (a female), whereas substrains bred in-house were tested by a group of 5 animal handlers including males and females. At least two studies have established that experimenter effects (Chesler, Wilson et al. 2002) and even the sex of the individual testing the mice (Sorge, Martin et al. 2014) can significantly affect the outcome of behavioral tests.

The gut microbiome has been implicated in numerous behavioral traits including locomotor response to psychostimulants (Kiraly, Walker et al. 2016, Meckel and Kiraly 2019). Composition of the gut microbiota, even in the same inbred strain background, can vary across time, from vendor to vendor, and even between different facilities and animal holding rooms at the same vendor or institution (Servick 2016, Ericsson and Franklin 2021). These differences can be attributed to a host of environmental factors including diet, caging, bedding and water supply (Lundberg, Bahl et al. 2017,

412 Bidot, Ericsson et al. 2018, Ericsson, Gagliardi et al. 2018). Host genetic background also plays a 413 significant role in the composition of the gut microbiota (Bubier, Chesler et al. 2021). Profound or even subtle changes in the gut microbiota in response to relocation from vendors to our vivarium could interact with different genetic backgrounds to significantly impact behavior. The relationship between genetic background and behavior becomes even more complicated when one considers that substrain behaviors attributable to stable differences in the gut microbiota could be erroneously ascribed solely to genetics. Shifts in the gut microbiota in response to changing environments could alter phenotypes and impact replicability from study to study. Recent studies have also established that the maternal 
microbiome can affect offspring neurodevelopment and impact behavior in adulthood (Codagnone, Stanton et al. 2019, Warner 2019, Vuong, Pronovost et al. 2020). Thus, it is important to consider not only the source of the mice being tested, but the composition of the maternal microbiome during neurodevelopment.

In summary, this study expands the knowledge of phenotypic differences in locomotor activity and initial response to cocaine in 6 sets of inbred mouse substrains which had previously not been characterized. Four of the six strain lineages displayed significant substrain differences in either basalor cocaine-induced locomotor behavior and can be utilized in RCCs to identify causal genetic variants. Environmental factors also warrant follow-up, as differences in behavior were observed across the same inbred substrains obtained from different sources. $\mathrm{C} 3 \mathrm{H} / \mathrm{He}$ and $\mathrm{DBA} / 2$ lineages demonstrated stable and robust differences in cocaine-induced locomotor behavior, and are good candidates for additional studies to investigate genetic and environmental factors that contribute to initial cocaine sensitivity. Future studies can utilize these data to increase our understanding of the complex factors that increase CUD and potentially lead to new therapeutic targets.

\section{References}

436 Bentzley, B. S., S. S. Han, S. Neuner, K. Humphreys, K. M. Kampman and C. H. Halpern (2021).

"Comparison of Treatments for Cocaine Use Disorder Among Adults: A Systematic Review and Meta-analysis." JAMA Netw Open 4(5): e218049.

439 Bidot, W. A., A. C. Ericsson and C. L. Franklin (2018). "Effects of water decontamination methods and bedding material on the gut microbiota." PLoS One 13(10): e0198305.

441 Boyle, A. E. and K. Gill (2001). "Sensitivity of AXB/BXA recombinant inbred lines of mice to the 442 locomotor activating effects of cocaine: a quantitative trait loci analysis." Pharmacogenetics 11(3): $443255-264$.

444 Boyle, A. E. and K. J. Gill (2009). "A verification of previously identified QTLs for cocaine-induced activation using a panel of B6.A chromosome substitution strains (CSS) and A/J x C57B1/6J F2 mice." Psychopharmacology (Berl) 207(2): 325-334.

447 Bryant CD, F. M., PDMV Fernando, Damaj MI, Kumar V, Mulligan MK (2018). "Reduced 448 Complexity Cross Design for Behavioral Genetics

$449 \quad$. Academic Press(Molecular-Genetic and Statistical Techniques for Behavioral and Neural 450 Research): 165-190.

451 Bubier, J. A., E. J. Chesler and G. M. Weinstock (2021). "Host genetic control of gut microbiome 452 composition." Mamm Genome 32(4): 263-281.

453 Cabana-Dominguez, J., A. Shivalikanjli, N. Fernandez-Castillo and B. Cormand (2019). "Genome454 wide association meta-analysis of cocaine dependence: Shared genetics with comorbid conditions." 
456

457

458

459

460

461

462

463

464

465

466

467

468

469

470

471

472

473

474

475

476

477

478

479

480

481

482

483

484

485

486

487

488

489

490

491

492

493

494

495

Chesler, E. J., S. G. Wilson, W. R. Lariviere, S. L. Rodriguez-Zas and J. S. Mogil (2002).

"Identification and ranking of genetic and laboratory environment factors influencing a behavioral trait, thermal nociception, via computational analysis of a large data archive." Neurosci Biobehav Rev 26(8): 907-923.

Chesler, E. J., S. G. Wilson, W. R. Lariviere, S. L. Rodriguez-Zas and J. S. Mogil (2002). "Influences of laboratory environment on behavior." Nat Neurosci 5(11): 1101-1102.

Codagnone, M. G., C. Stanton, S. M. O'Mahony, T. G. Dinan and J. F. Cryan (2019). "Microbiota and Neurodevelopmental Trajectories: Role of Maternal and Early-Life Nutrition." Ann Nutr Metab 74 Suppl 2: 16-27.

Crabbe, J. C., D. Wahlsten and B. C. Dudek (1999). "Genetics of mouse behavior: interactions with laboratory environment." Science 284(5420): 1670-1672.

Davidson, E. S., J. F. Finch and S. Schenk (1993). "Variability in subjective responses to cocaine: initial experiences of college students." Addict Behav 18(4): 445-453.

de Wit, H. and T. J. Phillips (2012). "Do initial responses to drugs predict future use or abuse?" Neurosci Biobehav Rev 36(6): 1565-1576.

Dickson, P. E., M. M. Miller, M. A. Calton, J. A. Bubier, M. N. Cook, D. Goldowitz, E. J. Chesler and G. Mittleman (2016). "Systems genetics of intravenous cocaine self-administration in the BXD recombinant inbred mouse panel." Psychopharmacology (Berl) 233(4): 701-714.

Ducci, F. and D. Goldman (2012). "The genetic basis of addictive disorders." Psychiatr Clin North Am 35(2): 495-519.

Ericsson, A. C. and C. L. Franklin (2021). "The gut microbiome of laboratory mice: considerations and best practices for translational research." Mammalian Genome.

Ericsson, A. C., J. Gagliardi, D. Bouhan, W. G. Spollen, S. A. Givan and C. L. Franklin (2018). "The influence of caging, bedding, and diet on the composition of the microbiota in different regions of the mouse gut." Sci Rep 8(1): 4065.

Gelernter, J., R. Sherva, R. Koesterer, L. Almasy, H. Zhao, H. R. Kranzler and L. Farrer (2014).

"Genome-wide association study of cocaine dependence and related traits: FAM53B identified as a risk gene." Mol Psychiatry 19(6): 717-723.

Gill, K. J. and A. E. Boyle (2003). "Confirmation of quantitative trait loci for cocaine-induced activation in the $\mathrm{AcB} / \mathrm{BcA}$ series of recombinant congenic strains." Pharmacogenetics 13(6): 329338.

Goldman, D., G. Oroszi and F. Ducci (2005). "The genetics of addictions: uncovering the genes." Nat Rev Genet 6(7): 521-532.

Haertzen, C. A., T. R. Kocher and K. Miyasato (1983). "Reinforcements from the first drug experience can predict later drug habits and/or addiction: results with coffee, cigarettes, alcohol, barbiturates, minor and major tranquilizers, stimulants, marijuana, hallucinogens, heroin, opiates and cocaine." Drug Alcohol Depend 11(2): 147-165.

Jalal, H., J. M. Buchanich, M. S. Roberts, L. C. Balmert, K. Zhang and D. S. Burke (2018).

"Changing dynamics of the drug overdose epidemic in the United States from 1979 through 2016."

Science 361(6408). 

Erwin (1999). "Quantitative-trait loci analysis of cocaine-related behaviours and neurochemistry." Pharmacogenetics 9(5): 607-617.

499 Karila, L., A. Petit, W. Lowenstein and M. Reynaud (2012). "Diagnosis and consequences of cocaine 500 addiction." Curr Med Chem 19(33): 5612-5618.

501 Kiraly, D. D., D. M. Walker, E. S. Calipari, B. Labonte, O. Issler, C. J. Pena, E. A. Ribeiro, S. J.

502 Russo and E. J. Nestler (2016). "Alterations of the Host Microbiome Affect Behavioral Responses to 503 Cocaine." Sci Rep 6: 35455.

504 Kirkpatrick, S. L., L. R. Goldberg, N. Yazdani, R. K. Babbs, J. Wu, E. R. Reed, D. F. Jenkins, A. F. 505 Bolgioni, K. I. Landaverde, K. P. Luttik, K. S. Mitchell, V. Kumar, W. E. Johnson, M. K. Mulligan, 506 P. Cottone and C. D. Bryant (2017). "Cytoplasmic FMR1-Interacting Protein 2 Is a Major Genetic 507 Factor Underlying Binge Eating." Biol Psychiatry 81(9): 757-769.

508 Kumar, V., K. Kim, C. Joseph, S. Kourrich, S. H. Yoo, H. C. Huang, M. H. Vitaterna, F. P. de 509 Villena, G. Churchill, A. Bonci and J. S. Takahashi (2013). "C57BL/6N mutation in cytoplasmic 510 FMRP interacting protein 2 regulates cocaine response." Science 342(6165): 1508-1512.

511 Lambert, N. M., M. McLeod and S. Schenk (2006). "Subjective responses to initial experience with 512 cocaine: an exploration of the incentive-sensitization theory of drug abuse." Addiction 101(5): 713513725.

514 Lundberg, R., M. I. Bahl, T. R. Licht, M. F. Toft and A. K. Hansen (2017). "Microbiota composition 515 of simultaneously colonized mice housed under either a gnotobiotic isolator or individually ventilated 516 cage regime." Sci Rep 7: 42245.

517 Meckel, K. R. and D. D. Kiraly (2019). "A potential role for the gut microbiome in substance use 518 disorders." Psychopharmacology (Berl) 236(5): 1513-1530.

519 Mennis, J., G. J. Stahler and M. J. Mason (2016). "Risky Substance Use Environments and 520 Addiction: A New Frontier for Environmental Justice Research." Int J Environ Res Public Health 521 13(6).

522 Merikangas, K. R. and S. Avenevoli (2000). "Implications of genetic epidemiology for the prevention of substance use disorders." Addict Behav 25(6): 807-820.

Miner, L. L. and R. J. Marley (1995). "Chromosomal mapping of the psychomotor stimulant effects of cocaine in BXD recombinant inbred mice." Psychopharmacology (Berl) 122(3): 209-214. Lariviere, D. B. Matthews, G. Mittleman, D. Goldowitz and E. J. Chesler (2010). "High-throughput behavioral phenotyping in the expanded panel of BXD recombinant inbred strains." Genes Brain Behav 9(2): 129-159.

530 Phillips, T. J., M. G. Huson and C. S. McKinnon (1998). "Localization of genes mediating acute and 531 sensitized locomotor responses to cocaine in BXD/Ty recombinant inbred mice." J Neurosci 18(8): 532 3023-3034.

533 SAMHSA (2019). "Key substance use and mental health indicators in the United States: Results from 534 the 2019 National Survey on Drug Use and Health." Substance Abuse and Mental Health Services 535 Administration.

536 Servick, K. (2016). "Of mice and microbes." Science 353(6301): 741-743. 
Sigmon, J. S., M. W. Blanchard, R. S. Baric, T. A. Bell, J. Brennan, G. A. Brockmann, A. W. Burks, J. M. Calabrese, K. M. Caron, R. E. Cheney, D. Ciavatta, F. Conlon, D. B. Darr, J. Faber, C. Franklin, T. R. Gershon, L. Gralinski, B. Gu, C. H. Gaines, R. S. Hagan, E. G. Heimsath, M. T. Heise, P. Hock, F. Ideraabdullah, J. C. Jennette, T. Kafri, A. Kashfeen, M. Kulis, V. Kumar, C. Linnertz, A. Livraghi-Butrico, K. C. K. Lloyd, C. Lutz, R. M. Lynch, T. Magnuson, G. K. Matsushima, R. McMullan, D. R. Miller, K. L. Mohlke, S. S. Moy, C. E. Y. Murphy, M. Najarian, L. O'Brien, A. A. Palmer, B. D. Philpot, S. H. Randell, L. Reinholdt, Y. Ren, S. Rockwood, A. R. Rogala, A. Saraswatula, C. M. Sassetti, J. C. Schisler, S. A. Schoenrock, G. D. Shaw, J. R. Shorter, C. M. Smith, C. L. St Pierre, L. M. Tarantino, D. W. Threadgill, W. Valdar, B. J. Vilen, K. Wardwell, J. K. Whitmire, L. Williams, M. J. Zylka, M. T. Ferris, L. McMillan and F. P. Manuel de Villena (2020). "Content and Performance of the MiniMUGA Genotyping Array: A New Tool To Improve Rigor and Reproducibility in Mouse Research." Genetics 216(4): 905-930.

Sorge, R. E., L. J. Martin, K. A. Isbester, S. G. Sotocinal, S. Rosen, A. H. Tuttle, J. S. Wieskopf, E. L. Acland, A. Dokova, B. Kadoura, P. Leger, J. C. Mapplebeck, M. McPhail, A. Delaney, G. Wigerblad, A. P. Schumann, T. Quinn, J. Frasnelli, C. I. Svensson, W. F. Sternberg and J. S. Mogil (2014). "Olfactory exposure to males, including men, causes stress and related analgesia in rodents." Nat Methods 11(6): 629-632.

554 Sullivan, P. F., A. Agrawal, C. M. Bulik, O. A. Andreassen, A. D. Borglum, G. Breen, S. Cichon, H. J. Edenberg, S. V. Faraone, J. Gelernter, C. A. Mathews, C. M. Nievergelt, J. W. Smoller, M. C. O'Donovan and C. Psychiatric Genomics (2018). "Psychiatric Genomics: An Update and an Agenda." Am J Psychiatry 175(1): 15-27.

558 Sun, J., H. R. Kranzler, J. Gelernter and J. Bi (2020). "A genome-wide association study of cocaine use disorder accounting for phenotypic heterogeneity and gene-environment interaction." $\underline{J}$

$560 \quad$ Psychiatry Neurosci 45(1): 34-44.

Tarantino, L. M., G. E. McClearn, L. A. Rodriguez and R. Plomin (1998). "Confirmation of quantitative trait loci for alcohol preference in mice." Alcohol Clin Exp Res 22(5): 1099-1105.

Thatcher, D. L. and D. B. Clark (2008). "Adolescents at risk for substance use disorders: role of psychological dysregulation, endophenotypes, and environmental influences." Alcohol Res Health 31(2): 168-176.

Thomsen, M. and S. B. Caine (2011). "Psychomotor stimulant effects of cocaine in rats and 15 mouse strains." Exp Clin Psychopharmacol 19(5): 321-341.

Tolliver, B. K., J. K. Belknap, W. E. Woods and J. M. Carney (1994). "Genetic analysis of sensitization and tolerance to cocaine." J Pharmacol Exp Ther 270(3): 1230-1238. C. J. Wilson, T. Rendon and E. Y. Hsiao (2020). "The maternal microbiome modulates fetal neurodevelopment in mice." Nature 586(7828): 281-286.

573 Wahlsten, D., P. Metten, T. J. Phillips, S. L. Boehm, 2nd, S. Burkhart-Kasch, J. Dorow, S. Doerksen, Neurobiol 54(1): 283-311.

578 Warner, B. B. (2019). "The contribution of the gut microbiome to neurodevelopment and 

strains." Genes Brain Behav 14(3): 271-280.

Figure 1. Acute cocaine induced locomotor activity for vendor supplied (not cohoused) and bred in-house (cohoused) A substrains. (A) Total distance moved in $30 \mathrm{mins}$ for each test day for vendor supplied female and male A substrains. While not significant, there was an overall increase in cocaine induced locomotor activity in the $\mathrm{A} / \mathrm{JCr}$ and $\mathrm{A} / \mathrm{JOlaHsd}$ substrains. (B) Total distance moved in 30 mins for each test day for bred in-house female and male A substrains. For both A substrains there was a decrease in locomotor activity across test days for male and female mice. Male mice were significantly more active compared to female mice. Each data point represents an individual mouse and error bars are standard error of the mean.

Figure 2. Acute cocaine induced locomotor activity for vendor supplied BALB/c substrains. Total distance moved in 30 mins for each test day for vendor supplied female and male BALB/c substrains. Across all test days female mice had significantly higher locomotor activity compared to male mice. Each data point represents an individual mouse and error bars are standard error of the mean. Total distance moved in 30-mins across each test day for cohoused female and male $\mathrm{C} 3 \mathrm{H}$ mice. While there were no sex differences, $\mathrm{C} 3 \mathrm{H} / \mathrm{HeNTac}$ male and female mice had higher locomotor activity and cocaine induced locomotor activity compared to $\mathrm{C} 3 \mathrm{H} / \mathrm{HeJ}$ mice. Both substrains, regardless of sex, had significantly higher activity following cocaine exposure on day 3 compared to days 1 and 2. (B) Total distance moved in 30-mins across each test day for non-cohoused female and male $\mathrm{C} 3 \mathrm{H}$ mice. Male and female $\mathrm{C} 3 \mathrm{H} / \mathrm{HeNTac}$ mice had higher locomotor activity and cocaine induced locomotor activity compared to the other three substrains. Each data point represents an individual mouse and error bars

609 Figure 4. Acute cocaine induced locomotor activity for DBA/2 substrains that were vendor are standard error of the mean.

618 Figure 5. Acute cocaine induced locomotor activity for vendor supplied and bred in-house 619 FVB/N substrains. (A) Total distance moved in 30-mins for each test day for vendor supplied female 620 and male FVB/N substrains. All FVB/N substrains, regardless of sex, displayed significantly higher 621 locomotor activity following cocaine administration on day 3. (B) Total distance moved in 30 mins for 
622 each test day for female and male FVB/N substrains bred in-house and cohoused at weaning. Overall, 623 FVB/NTac male mice had significantly higher locomotor activity compared to FVB/NJ male mice. $624 \mathrm{FVB} / \mathrm{NJ}$ female mice had higher cocaine induced locomotor activity compared to FVB/NTac female 625 mice. Each data point represents an individual mouse and error bars are standard error of the mean.

626 Figure 6. Acute cocaine induced locomotor activity for NOD substrains bred in-house. Total 627 distance moved in 30 mins for each test day for female and male NOD substrains bred in-house. All 628 mice, regardless of substrain, had higher locomotor activity following cocaine administration 629 compared to saline. Each data point represents an individual mouse and error bars are standard error 630 of the mean.

631

632

633 


\section{(A) Vendor Supplied}
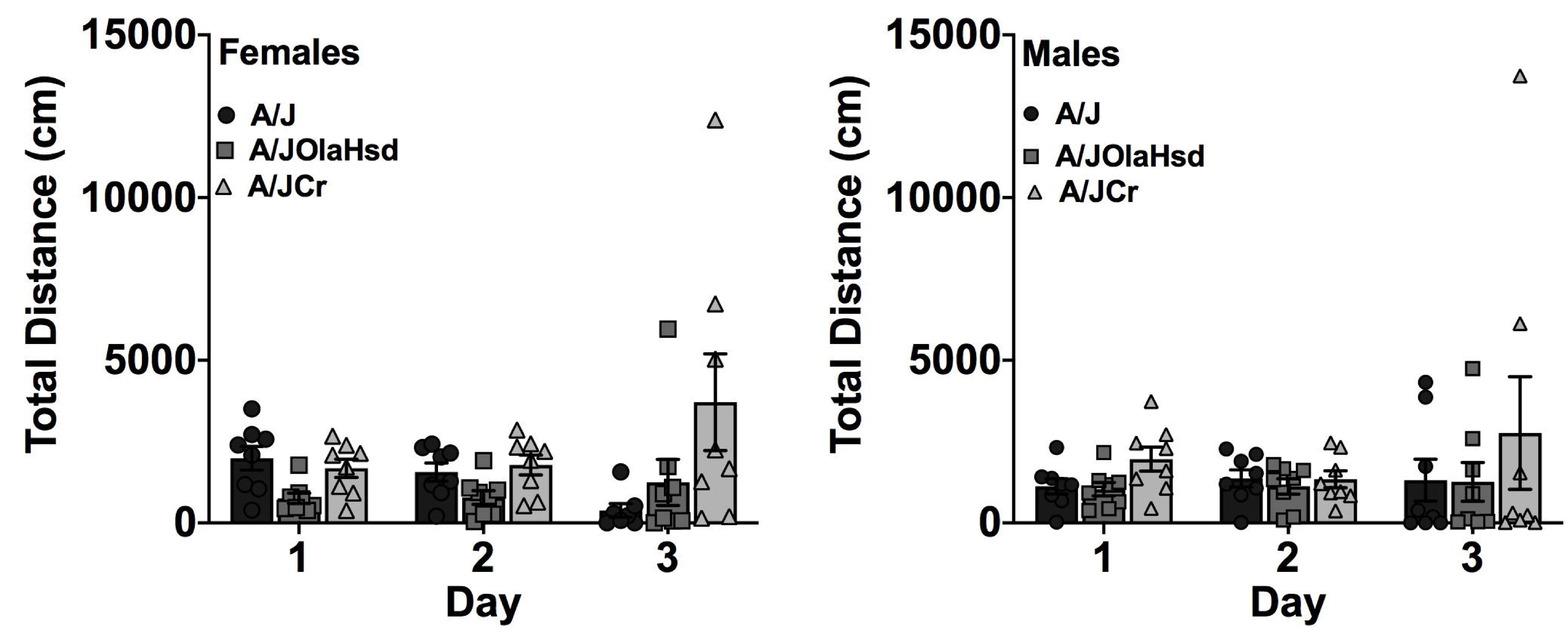

(B) Bred In-house
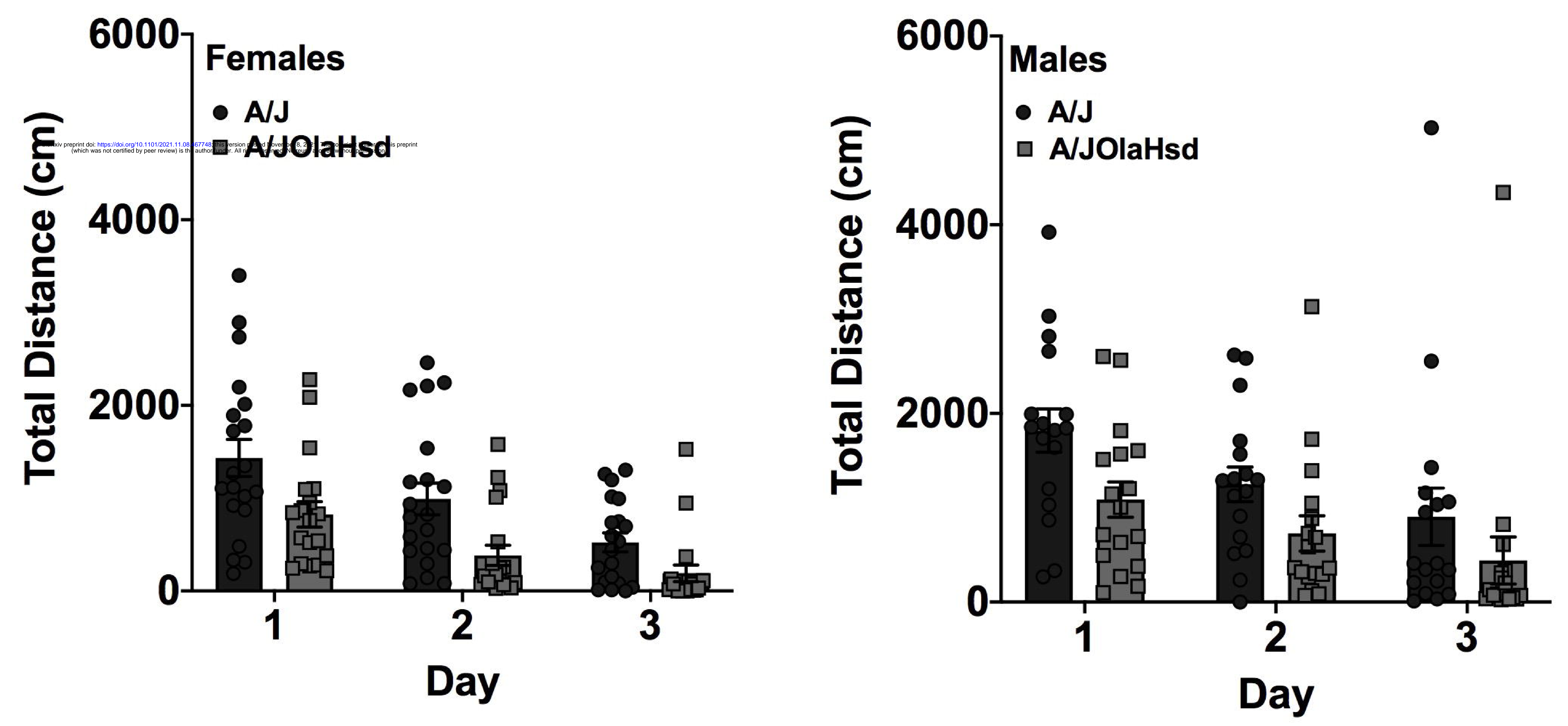


\section{(A) Vendor Supplied}
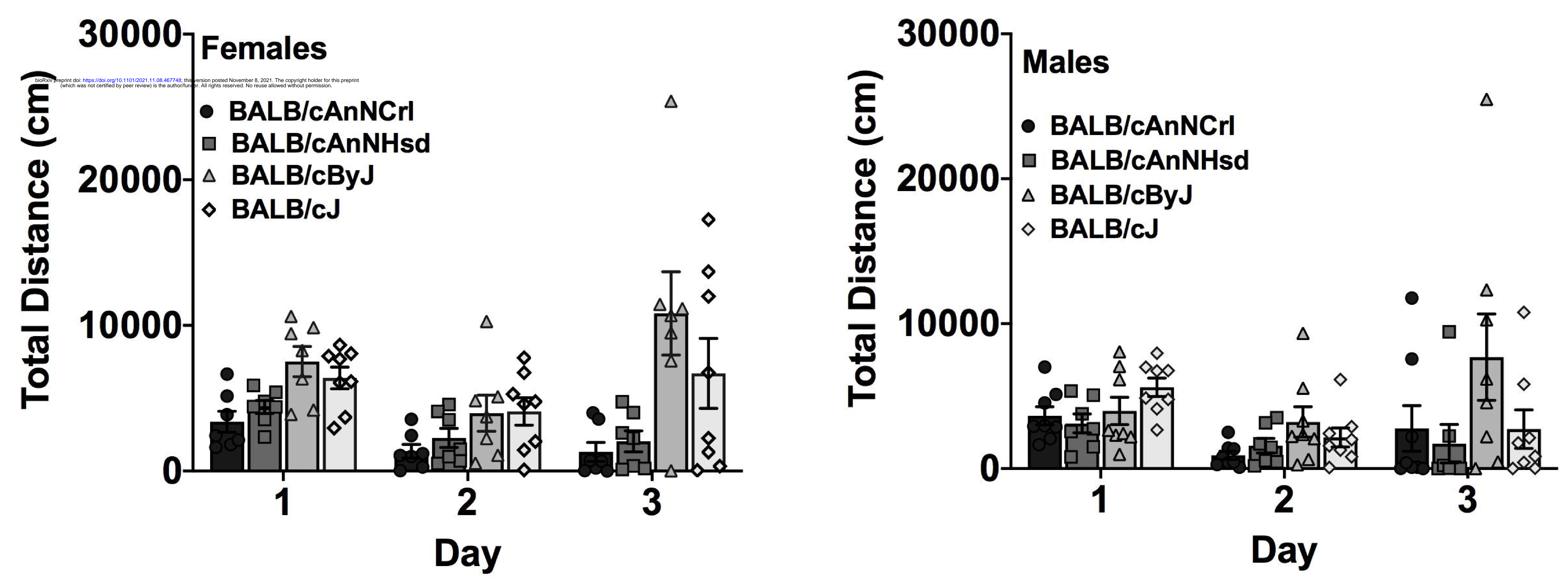


\section{(A) Vendor Supplied}
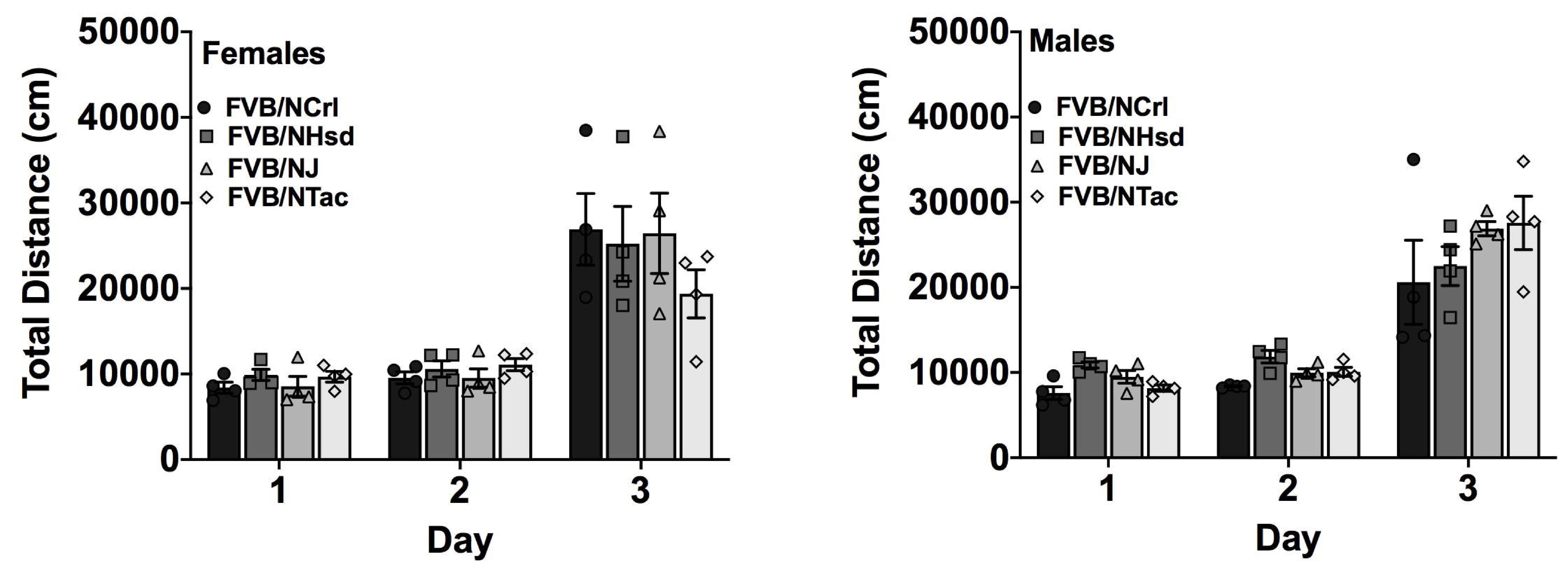

(B) Bred In-house
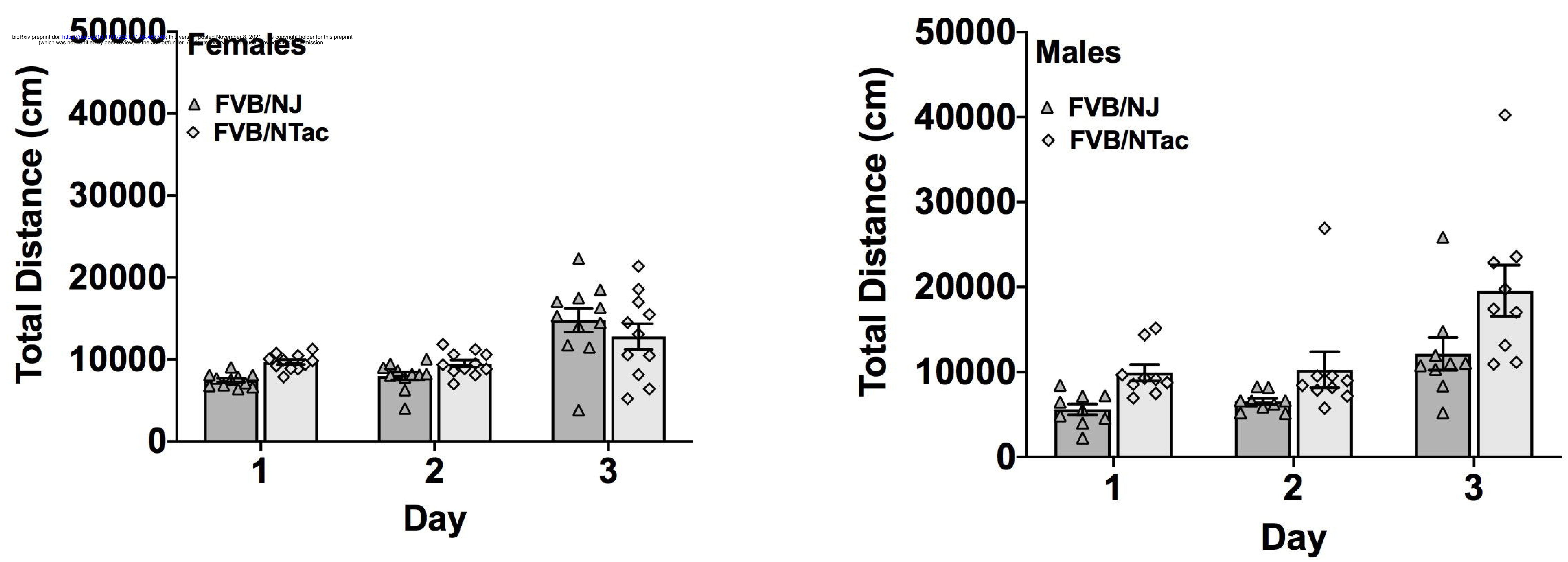
(A) Bred In-house
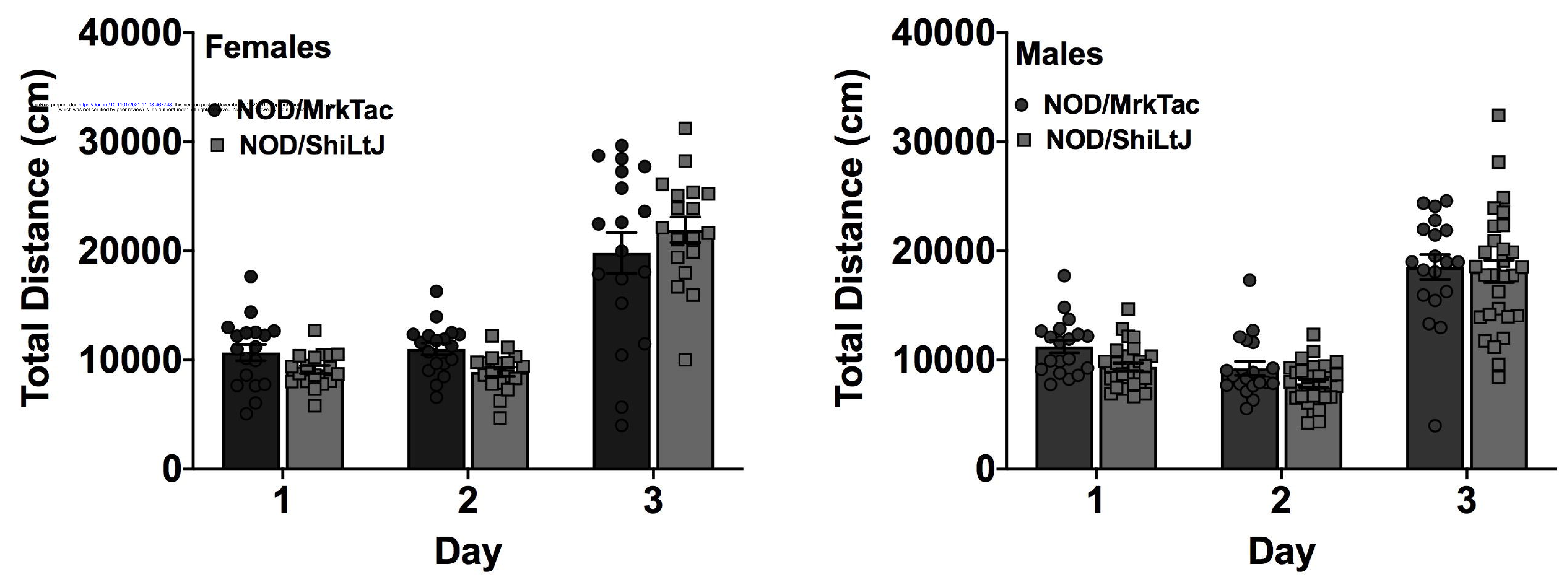